\title{
Determination of the magnetic structure of manganese carbodiimide with diffraction experiments using polarized neutrons
}

\author{
Manuel Krott, ${ }^{1}$ Andreas Houben, ${ }^{1}$ Paul Müller, ${ }^{1}$ Werner Schweika, ${ }^{2}$ and Richard Dronskowski ${ }^{1, *}$ \\ ${ }^{1}$ Institute of Inorganic Chemistry, RWTH Aachen University, D-52056 Aachen, Germany \\ ${ }^{2}$ Institut für Festkörperforschung, Streumethoden, Forschungszentrum Jülich, D-52428 Jülich, Germany \\ (Received 29 April 2009; revised manuscript received 24 June 2009; published 29 July 2009)
}

\begin{abstract}
The magnetic structure of $\mathrm{MnNCN}$, the nitrogen equivalent of $\mathrm{MnO}$, and the first carbodiimide of a non- $d^{10}$ transition metal ever made, has been determined on the basis of spin-polarized neutron-diffraction data close to absolute zero temperature. The $S=5 / 2$ moments of the high-spin $\mathrm{Mn}^{2+}$ ions lie within the $a b$ hexagonal sheets and exhibit an antiferromagnetic ordering between the sheets along the $c$ axis. Below the Néel point $(28 \mathrm{~K})$, a temperature-dependent ordering pattern exists due to magnetic frustration inside the sheets, characterized by a varying angle between the moments. A comparison with other magnetic structures reveals that the compact $\mathrm{NCN}^{2-}$ group is capable of mediating significant spin density needed to ensure relatively strong magnetic communication in view of a large $\mathrm{Mn}^{2+}-\mathrm{Mn}^{2+}$ separation.
\end{abstract}

DOI: 10.1103/PhysRevB.80.024117

PACS number(s): 61.05.fm, 77.84.Bw, 75.50.Ee

\section{INTRODUCTION}

Developing synthetic routes for novel magnetic compounds is an everlasting challenge in inorganic solid-state chemistry. In order to succeed, coupling of magnetically active centers such as $d$ - and $f$-configured ions with traditional bridging ligands, i.e., oxide, chalcogenide, or cyanide anions, has been pursued for many decades. As an alternative, the use of the corresponding base of $\mathrm{H}_{2} \mathrm{NCN}$, the $\mathrm{NCN}^{2-}$ anion which is either named carbodiimide ( $D_{\infty}$ symmetry, as in ${ }^{-} \mathrm{N}=\mathrm{C}=\mathrm{N}^{-}$with two double bonds) or cyanamide (potentially bent, as in $\mathrm{N} \equiv \mathrm{C}-\mathrm{N}^{2-}$ with one triple and one single bond), has recently come to a broader attention. Because of its two minus charge and the fact that $\mathrm{NCN}^{2-}$ is electronically dominated by the terminal nitrogen atoms, $\mathrm{NCN}^{2-}$ may be regarded a "divalent nitride" anion, thereby allowing us to repeat the entire oxide $\left(\mathrm{O}^{2-}\right)$ chemistry using a nitrogen species.

A good number of inorganic carbodiimides/cyanamides has been synthesized containing alkali ${ }^{1-5}$ and alkaline-earth metals, ${ }^{6-9}$ main-group elements, ${ }^{10-14} \quad d^{10}$ transition metals, ${ }^{15-21}$ and also rare-earth metals. ${ }^{22-25}$ In 2005 , the existence of quasibinary compounds containing non- $d^{10}$ magnetically active metals was first predicted using densityfunctional theory, indicating that all $M \mathrm{NCN}(M=\mathrm{Mn}, \mathrm{Fe}$, $\mathrm{Co}, \mathrm{Ni}$, and $\mathrm{Cu}$ ) phases should be unstable with respect to both formation enthalpy $\Delta H_{\mathrm{f}}$ and Gibbs formation energy $\Delta G_{\mathrm{f} .}{ }^{26}$ In the meantime, all five synthetic targets have been synthesized, for example, through a metathesis route for $\mathrm{MnNCN},{ }^{27}$ by the thermal decomposition of hydrogencontaining precursors for $M \mathrm{NCN}(M=\mathrm{Fe}, \mathrm{Co}$, and $\mathrm{Ni})$ (Refs. 28 and 29) and by the room-temperature oxidation of a $\mathrm{Cu}(\mathrm{I})$ precursor for CuNCN. ${ }^{30}$

Despite its rhombohedral symmetry, the crystal structure of MnNCN (Fig. 1, most conveniently described using a hexagonal setting) resembles the rock-salt motif because of octahedral coordination for both $\mathrm{Mn}^{2+}$ and $\mathrm{NCN}^{2-}$. Magneticsusceptibility measurements on this first carbodiimide of a magnetic transition metal ever made yielded antiferromagnetic interactions, a Néel point of about $28 \mathrm{~K}$ and a Curie-
Weiss behavior above that temperature; the magnetic moment was determined as $\mu=5.84 \mu_{\mathrm{B}}$ from the slope of the Curie-Weiss straight line, ${ }^{27}$ in accord with a high-spin $\mathrm{Mn}^{2+}$ ion (five unpaired electrons, $S=5 / 2$ ) and an expected moment of $\mu=g \sqrt{S(S+1)} \mu_{\mathrm{B}}=5.92 \mu_{\mathrm{B}} \cdot{ }^{31}$ In order to eventually

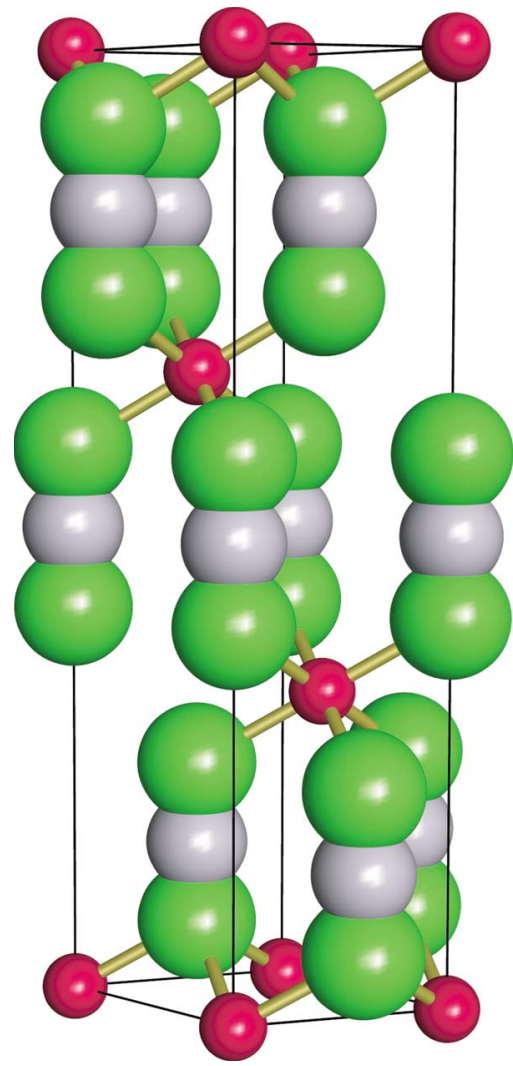

FIG. 1. (Color online) The hexagonal setting of the rhombohedral crystal structure of manganese carbodiimide, $\mathrm{MnNCN}$, with the $\mathrm{Mn}$ atoms given in red, $\mathrm{N}$ atoms in green, and $\mathrm{C}$ atoms in gray. The strictly linear $\mathrm{NCN}^{2-}$ carbodiimide groups coincide with the $c$ axis and coordinate the $\mathrm{Mn}$ atoms through their terminal $\mathrm{N}$ atoms to yield nearly regular octahedra with $6 \times \mathrm{Mn}-\mathrm{N}=2.26 \AA$ and $\angle(\mathrm{N}, \mathrm{Mn}, \mathrm{N})=84.1^{\circ}$ and $95.9^{\circ}$. 
disclose the magnetic structure of the nitrogen equivalent of manganese oxide, neutron powder-diffraction measurements have been carried out, both with and without spin-polarized neutrons.

\section{EXPERIMENT}

Samples of green-colored transparent powderous manganese carbodiimide were prepared via a metathesis reaction between $\mathrm{MnCl}_{2}$ and $\mathrm{ZnNCN}$ to yield $\mathrm{MnNCN}$ and $\mathrm{ZnCl}_{2}$ in quartz ampoules at $650{ }^{\circ} \mathrm{C} .{ }^{27}$ Single-crystal data confirmed MnNCN to exhibit rhombohedral symmetry (space group $R \overline{3} m$ ) with the hexagonal lattice parameters $a$ $=3.3583(4) \AA$ and $c=14.247(2) \AA$ at room temperature; the atoms are located on the Wyckoff sites $3 a(\mathrm{Mn}), 3 b(\mathrm{C})$, and $6 b \quad(\mathrm{~N})$ with $z=0.5855(3)$. The first neutron powderdiffraction data of MnNCN were collected at Forschungszentrum Jülich by means of the SV7 diffractometer with $\lambda$ $=1.0952(4) \AA$ in the $2 \theta$ range $4^{\circ} \leq 2 \theta \leq 90^{\circ}$ at temperatures of $T=4.5$ and $10 \mathrm{~K}$, between $T=15$ and $35 \mathrm{~K}$ in steps of $2 \mathrm{~K}$, and finally at room temperature. In addition, neutron measurements with spin-polarization analysis were carried out at FRM II, Garching, with the help of the most advanced diffuse neutron-scattering (DNS) instrument and $\lambda=4.74 \AA$ in the $2 \theta$ range $5^{\circ} \leq 2 \theta \leq 105^{\circ}$ at temperatures of $T=4.5$ and $20 \mathrm{~K}$. Although DNS typically serves as a "cold" time-offlight spectrometer, its use in diffraction mode (with a constant wavelength) but with polarization analysis served the problem of magnetic structure determination quite well. All numerical Rietveld refinements were carried out using the FULLPROF program suite. ${ }^{32}$

\section{RESULTS}

The nonpolarized neutron data collected at the SV7 instrument already corroborated the existence of an antiferromagnetic ordering below $28 \mathrm{~K}$ (Fig. 2, top) with a complicated temperature-dependent behavior that came quite unexpected. In Fig. 3, this phenomenon is more carefully analyzed for the case of the first two SV7 magnetic reflections. Figure 3 also allows a visual comparison with the previously published magnetic-susceptibility data. ${ }^{27}$ In particular, cooling below $30 \mathrm{~K}$ causes a strong rise in intensity for the first reflection (top) while the second one (middle) is hardly affected. Below $20 \mathrm{~K}$, however, the second peak increases considerably whereas the first one slightly weakens. Indeed, the magnetic-susceptibility mirrors that temperature dependence, highlighted by straight lines (Fig. 3, bottom) which are simple guides to the eye. Obviously, a set of different magnetic orderings exists. Nonetheless, because of insufficient statistics at the SV7 instrument, a definite structure model for the spin structure could not be developed in the very beginning. Thus, additional polarized neutron data had to be collected using the DNS instrument, and these yielded six well-shaped magnetic reflections (see Fig. 2, bottom) below the Néel point, two of which overlap substantially.

These six reflections could be indexed by doubling the unit cell in all directions. The first reflection was indexed as (003), hence the orientation of the spin moments can be ex-
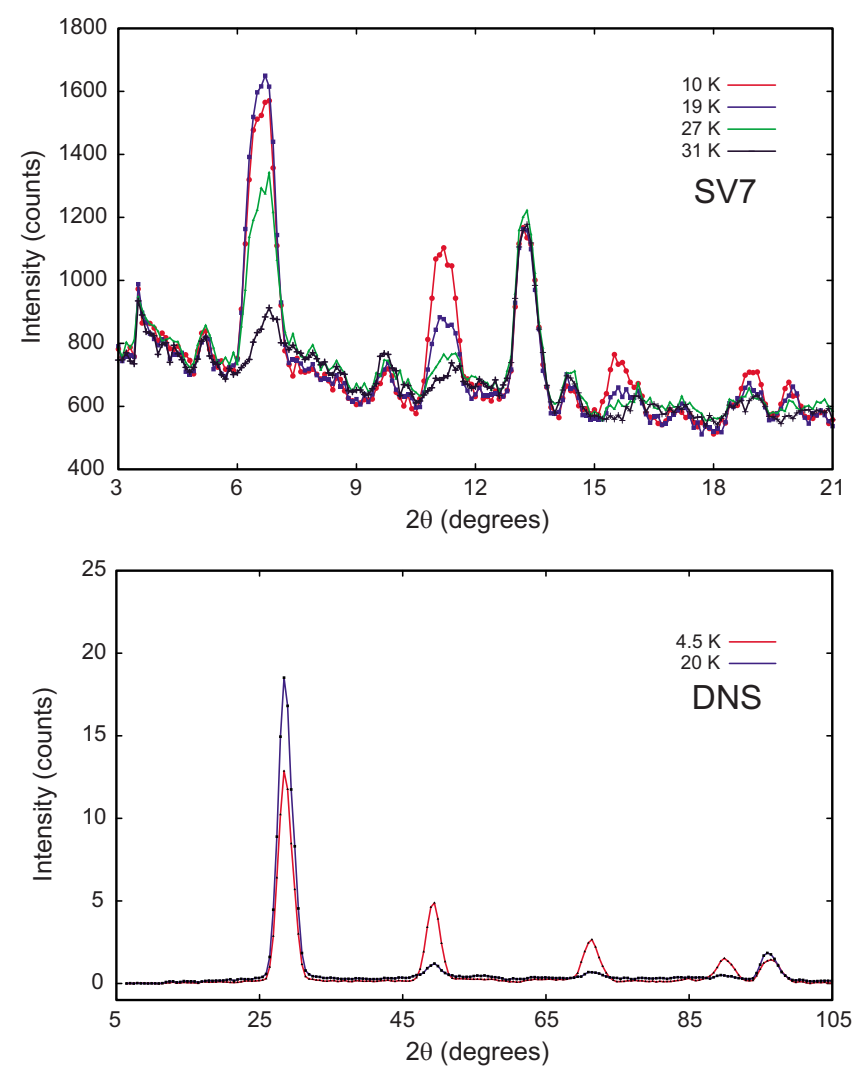

FIG. 2. (Color online) Neutron diffraction of MnNCN measured at the Jülich SV7 instrument (top, $\lambda=1.0952 \AA$ ) at four temperatures and the pure magnetic scattering as measured by polarization analysis at the Garching DNS instrument (bottom, $\lambda=4.74 \AA$ ) for $T=4.5$ and $20 \mathrm{~K}$.

cluded to point along the $c$ axis; consequently, the rhombohedral symmetry is entirely destroyed. Instead, the magnetic indexing refers to a triclinic symmetry and all calculations concerning the magnetic structure were performed in space group $P \overline{1} .^{32}$ To do so, only the magnetic DNS reflections were refined using the lattice parameters, the Gaussian peak profile, and the angle between the spin moments $(S=5 / 2)$ as refinable parameters. The best accordance between the observed and calculated intensities is depicted in Fig. 4 and Table I contains all relevant Rietveld parameters. Table II offers the Bragg positions and indices of the magnetic reflections.

In addition to the aforementioned structural characterization, we independently determined the value of the $\mathrm{Mn}^{2+}$ magnetic moment by a simultaneous refinement of the magnetic and the crystallographic reflections on the basis of the SV7 data because such a procedure requires the intensities of the nuclear reflections for scaling purposes. Table III provides all relevant information and the graphical result is depicted in Fig. 5. The magnetic moment based on neutron data arrives at $4.72(15) \mu_{\mathrm{B}}$, to be compared with the expectation value of the free $\mathrm{Mn}^{2+}$ ion which is $\mu_{\mathrm{a}}=g S \mu_{\mathrm{B}}=5 \mu_{\mathrm{B}}(g$ $=2, S=5 / 2$ ) for the neutron case; both values are identical within two sigma. 

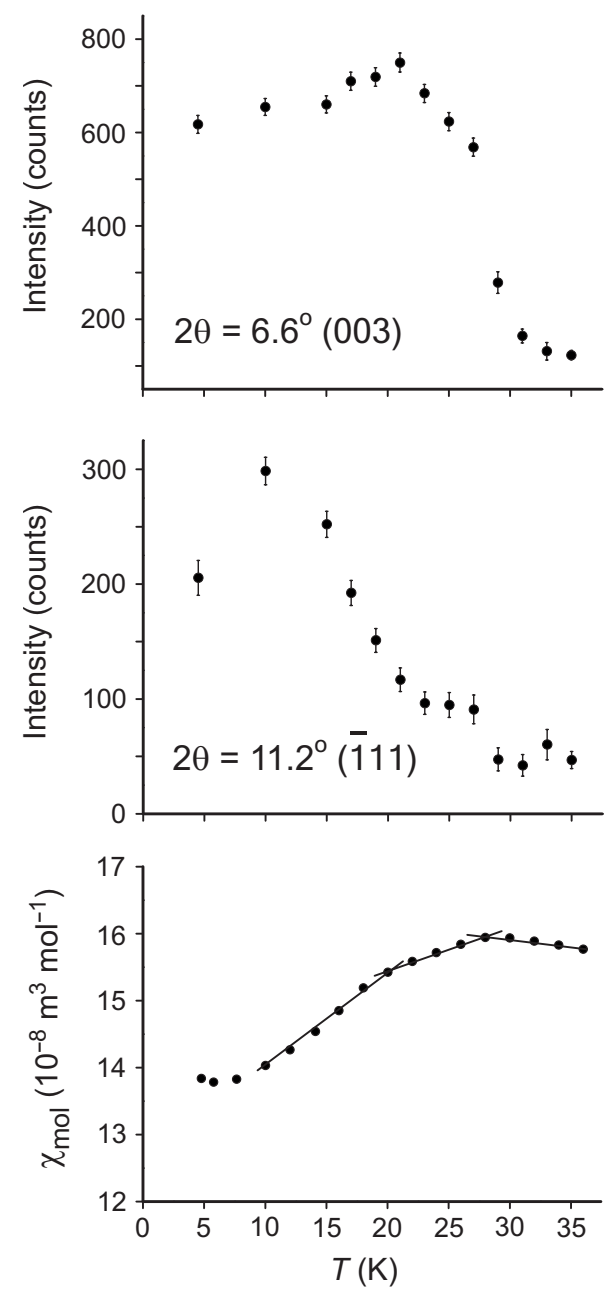

FIG. 3. Intensity changes of two SV7-measured magnetic reflections at $2 \theta=6.6^{\circ}$ (top) and at $2 \theta=11.2^{\circ}$ (middle) as a function of temperature; the course of the molar magnetic susceptibility (bottom) is given for comparison, together with trend lines as simple guides to the eye.

\section{DISCUSSION}

The magnetic structure(s) of MnNCN (Fig. 6) can be described by an antiferromagnetic ordering of the magnetic moments within the $a b$ sheets of the manganese ions stacked along the $c$ axis; despite the triclinic symmetry of the magnetic structure, we still refer to the hexagonal metric of the rhombohedral unit cell for reasons of simplicity. Inside these $a b$ sheets, the spins are orientated perpendicular to the $c$ axis and enclose an angle whose size depends on the temperature, thereby leading to the observed differences in intensities. Because all distances between the manganese ions in the sheets are equal, magnetic spin frustration seemingly occurs. With respect to a (hypothetical) ferromagnetic ordering within one sheet (i. e., $\varphi=0$ ), the spins are tilted against each other with an angle of $46^{\circ}$ at $20 \mathrm{~K}$ and $100.8^{\circ}$ at $4.5 \mathrm{~K}$. At $T=20 \mathrm{~K}$, the (003) reflection gains its maximum intensity, thereby also indicating maximum attainable precision for the magnetic structure; at the lowest measured temperature of $T=4.5 \mathrm{~K}$, the angle $\varphi$ widens. For a better understanding, Fig. 6 depicts the entire magnetic unit cells at $T=4.5$ and $20 \mathrm{~K}$. We also
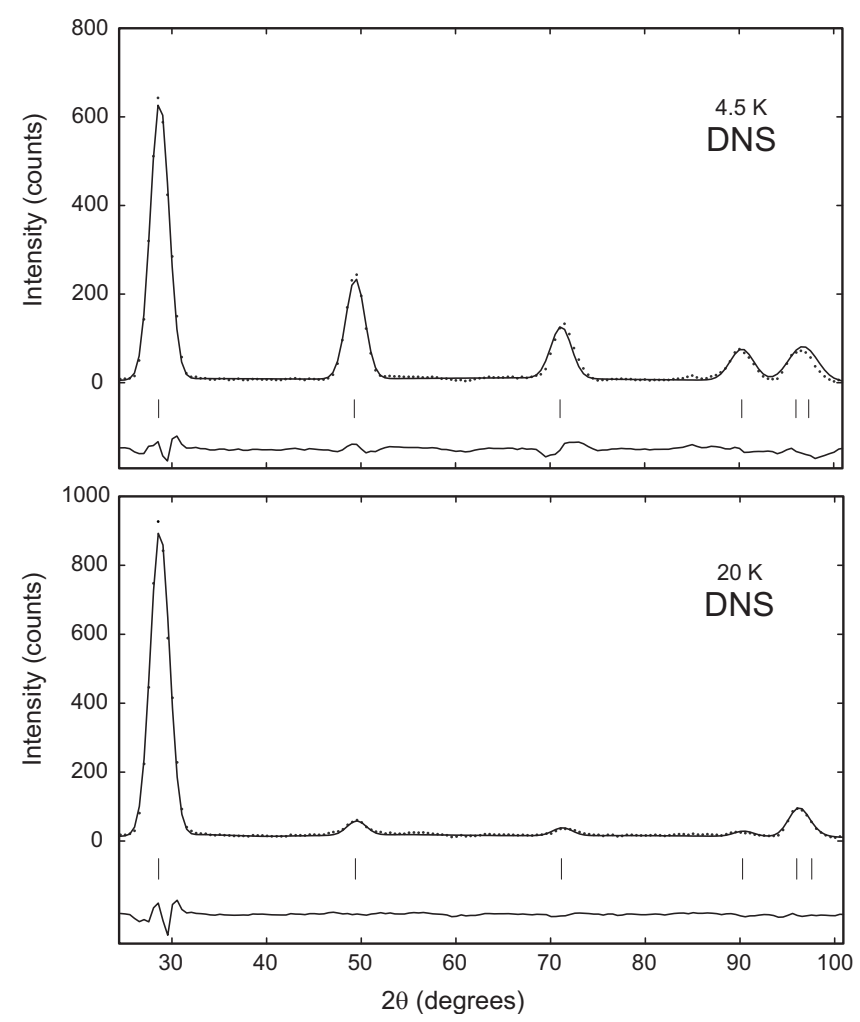

FIG. 4. Magnetic neutron reflections measured at the DNS instrument and their refined intensities as calculated with the Rietveld parameters given in Table II for $T=4.5$ (top) and $20 \mathrm{~K}$ (bottom). The positions of the Bragg peaks are marked by vertical dashes and the difference curve is given below.

note that the spin structure at $T=4.5 \mathrm{~K}$ (Fig. 6, top left) alludes to the possibility of a structural distortion but this is clearly beyond the resolution limit of our data.

To further understand the magnetic structure of MnNCN, a comparison with those seen in $\mathrm{MnO}$ and also the isostructural $\alpha$-MnS is appropriate. ${ }^{33,34}$ Note that the magnetic structure of $\mathrm{MnO}$ serves as the archetype, simply because (a) $\mathrm{MnO}$ is the oxide equivalent of $\mathrm{MnNCN}$ and (b) the $\mathrm{MnO}$ structure was the very first magnetic structure ever investigated and solved by neutron scattering. In 1951, Shull performed neutron-diffraction studies on $\mathrm{MnO}$ and suggested a

TABLE I. Structure and profile parameters for the refined neutron-diffraction data of MnNCN collected at the DNS instrument at temperatures of $T=4.5$ and $20 \mathrm{~K} . \varphi$ refers to the angle between two neighboring spin moments; for illustration, see also Fig. 6. Due to the limited number of reflections, $U, V$, and $W$ could not be refined simultaneously such that standard deviations cannot be specified for those values.

\begin{tabular}{lcc}
\hline \hline & $T=4.5 \mathrm{~K}$ & $T=20 \mathrm{~K}$ \\
\hline Lattice parameters: $(\AA)$ & $a=6.68(1)$ & $a=6.67(5)$ \\
$\varphi(\mathrm{deg})$ & $c=28.68(7)$ & $c=28.66(7)$ \\
$U, V, W$ & $100.8(4)$ & $46(2)$ \\
$R_{\mathrm{p}}, R_{\text {Bragg }}$ & $2.24,-2.15,2.72$ & $2.75,-0.34,2.48$ \\
\hline \hline
\end{tabular}


TABLE II. Bragg positions and indices of the magnetic reflections measured at DNS.

\begin{tabular}{|c|c|c|c|c|}
\hline \multicolumn{2}{|c|}{$T=4.5 \mathrm{~K}$} & \multicolumn{2}{|c|}{$T=20 \mathrm{~K}$} & \multirow[b]{2}{*}{$\begin{array}{r}\text { Index } \\
(h k l)\end{array}$} \\
\hline $\begin{array}{l}\text { Bragg position } \\
\text { in } 2 \theta \text { (deg) }\end{array}$ & Intensity & $\begin{array}{l}\text { Bragg position } \\
\text { in } 2 \theta \text { (deg) }\end{array}$ & Intensity & \\
\hline 28.711 & 1567 & 28.725 & 2247 & 003 \\
\hline 49.408 & 571 & 49.514 & 125 & $\overline{1} 11$ \\
\hline 71.167 & 331 & 71.271 & 61 & $1 \overline{15}$ \\
\hline 90.288 & 215 & 90.406 & 38 & $\overline{1} 17$ \\
\hline 96.113 & 123 & 96.176 & 205 & 009 \\
\hline 97.468 & 101 & 97.716 & 23 & 113 \\
\hline
\end{tabular}

structure model with a cell whose edge is twice the length of the crystallographic cell and which exhibits ferromagnetic sheets parallel to the (111) plane. Neighboring planes are coupled in an antiferromagnetic fashion and the magnetic moments were suggested to run parallel to the [001] axis. ${ }^{34}$ Seven years later, the model was revised by Roth, with a corrected ferromagnetic spin orientation lying within the (111) plane and this model is covered in many solid-state physics textbooks. The in-plane orientation of the magnetic moments with respect to the crystallographic axes, however, was indeterminable from powder data ${ }^{35}$ due to overlapping reflections because of the cubic symmetry. In 1988, a lowtemperature high-resolution neutron study indicated that the true symmetry of $\mathrm{MnO}$ at $8 \mathrm{~K}$ must be lower than rhombohedral. ${ }^{36}$ Total neutron-scattering data eventually yielded, in 2006, that the ferromagnetically aligned spins within the (111) sheets show a slight preference for spin alignment along the $\langle 11 \overline{2}\rangle$ directions. ${ }^{37}$

Contrasting this magnetic structure with our aforementioned model for MnNCN, one finds similarities as well as dissimilarities. For both $\mathrm{MnO}$ and $\mathrm{MnNCN}$, the magnetic structure can be described by ferromagnetic ( $\mathrm{MnO})$ and almost ferromagnetic (MnNCN) layers of $\mathrm{Mn}^{2+}$ ions which are stacked antiferromagnetically along the crystallographically threefold axis, namely, [111] for $\mathrm{MnO}$ and [001] for MnNCN. Nonetheless, the MnNCN structure contains one additional degree of freedom for the orientations of the spins. The ferromagnetic orientation within one sheet of $\mathrm{Mn}^{2+}$ ions corresponds to a constant $\varphi=0^{\circ}$ scenario $(\mathrm{MnO}$ case) between the magnetic moments. For MnNCN, how-

TABLE III. Structure and profile parameters for the refined neutron-diffraction data of MnNCN collected at the SV7 instrument at $T=4.5 \mathrm{~K}$

\begin{tabular}{lcc}
\hline \hline & Crystallographic & Magnetic \\
\hline Number of reflections: & 55 & 147 \\
Lattice parameters: $(\AA)$ & $a=3.329(3)$ & $a=6.66(1)$ \\
Magnetic moment: & $c=14.247(15)$ & $c=28.49(3)$ \\
$R_{\mathrm{p}}, R_{\text {Bragg }}$ & & $4.72(15) \mu_{\mathrm{B}}$ \\
\hline \hline
\end{tabular}

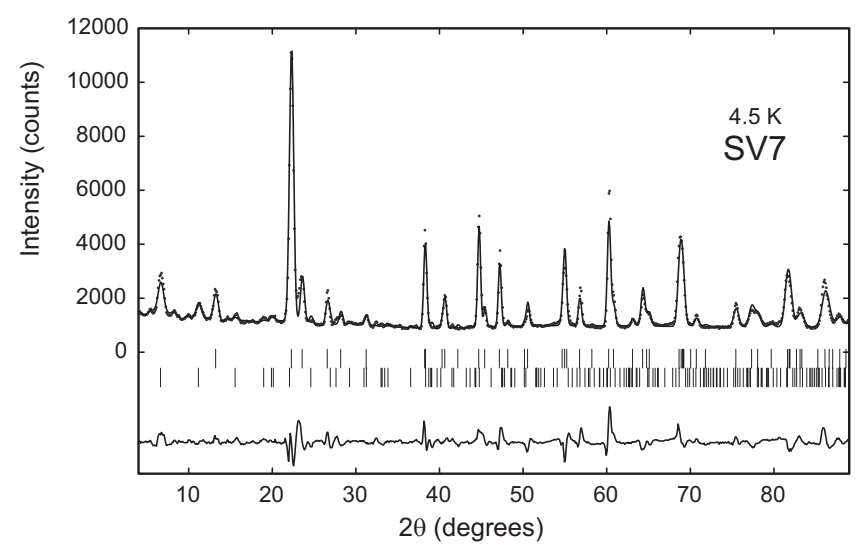

FIG. 5. Neutron-diffraction pattern of MNCN measured at the SV7 instrument at $T=4.5 \mathrm{~K}$ and its Rietveld refinement based on the parameters as given in Table III. The positions of the Bragg peaks are marked by vertical dashes both for the crystallographic (upper row) and the magnetic structure (lower row), and the difference curve is given below.

ever, this very angle is a complicated function of the temperature and the different behavior of $\mathrm{MnO}$ and $\mathrm{MnNCN}$ must be a consequence of unlike in-sheet $\mathrm{Mn}-\mathrm{Mn}$ distances (MnO: $3.13 \AA$; MnNCN: $3.36 \AA$ ). We also suspect that the spin orientation found for $\mathrm{MnNCN}$ resembles the one which has been observed for another (but less well-known) compound, $\mathrm{MnCO}_{3}{ }^{38}$

Comparing $T_{\mathrm{N}}$ of all phases, one finds 118(2) $\mathrm{K}$ for $\mathrm{MnO},{ }^{36} 152.0(8) \mathrm{K}$ for $\alpha-\mathrm{MnS},{ }^{33}$ and $31.5 \mathrm{~K}$ for $\mathrm{MnCO}_{3}$ (Ref. 39) such that the Néel temperature of MnNCN (28 K) (Ref. 27) is in the same region as $\mathrm{MnCO}_{3}$. At first sight, this looks remarkable because the distance between the layers of $\mathrm{Mn}^{2+}$ ions in MnNCN (about $4.7 \AA$ ) is almost twice the distance as in $\mathrm{MnCO}_{3}$ (about $2.6 \AA$ ). Clearly, the exchange interactions between the layers are similar although both anions differ remarkably in their shapes.
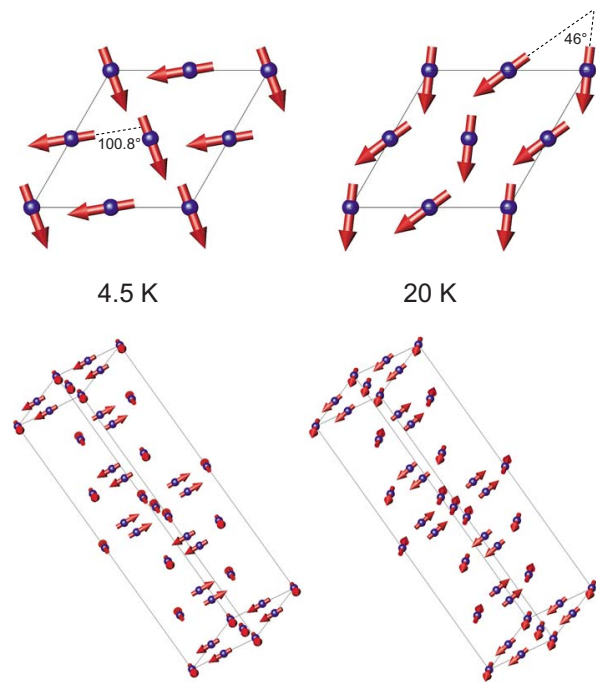

FIG. 6. (Color online) Arrangement of the spin moments in the $a b$ plane of $\mathrm{MnNCN}$ at $T=4.5 \mathrm{~K}$ (top left) and at $T=20 \mathrm{~K}$ (top right), together with the entire magnetic unit cell for $T=4.5 \mathrm{~K}$ (bottom left) and $T=20 \mathrm{~K}$ (bottom right). The angles between the neighboring magnetic moments have been indicated. 


\section{CONCLUSION}

At lowest temperatures, MnNCN, the nitrogen equivalent of $\mathrm{MnO}$, exhibits significantly different and temperaturedependent magnetic structures due to magnetic frustration in the $a b$ sheets of the rhombohedral structure. Thus, the phenomenon nicely fits into the picture of those magnetic lattices which are geometrically or topologically prone to frustration because of being based on layered triangular motifs. ${ }^{40}$ Similar to the case of $\mathrm{MnO}$, the spin layers are antiferromagnetically stacked on top of each other along the crystallographically threefold axis. The numerical data reveal that the carbodiimide group is capable to ensure strong magnetic coupling despite relatively wide $\mathrm{Mn}^{2+}-\mathrm{Mn}^{2+}$ distances between the sheets.

\section{ACKNOWLEDGMENTS}

It is a pleasure to thank J. Walter (SV7) and Y. Su (DNS) for having collected the neutron-diffraction data. This study was made possible through financial grants by Deutsche Forschungsgemeinschaft and Bundesminister für Bildung und Forschung whose support is also gratefully acknowledged.
*FAX: +49 (0) 241 80-92642; drons@ @al9000.ac.rwth-aachen.de

${ }^{1}$ M. G. Down, M. J. Haley, P. Hubberstey, R. J. Pulham, and A. E. Thunder, J. Chem. Soc. Dalton Trans. 1978, 1407.

${ }^{2}$ A. Harper and P. Hubberstey, J. Chem. Res., Synop. 7, 194 (1989).

${ }^{3}$ M. Becker and M. Jansen, Z. Anorg. Allg. Chem. 626, 2505 (2000).

${ }^{4}$ W. Schnick and H. Huppertz, Z. Anorg. Allg. Chem. 621, 1703 (1995).

${ }^{5}$ M. Becker and M. Jansen, Z. Naturforsch. 54b, 1375 (1999).

${ }^{6}$ N.-G. Vannerberg, Acta Chem. Scand. 16, 2263 (1962).

${ }^{7}$ U. Berger and W. Schnick, J. Alloys Compd. 206, 179 (1994).

${ }^{8}$ O. Reckeweg and F. J. DiSalvo, Angew. Chem., Int. Ed. 39, 412 (2000).

${ }^{9}$ W. Liao and R. Dronskowski, Acta Crystallogr. E 60, i124 (2004).

${ }^{10}$ R. Dronskowski, Z. Naturforsch. 50b, 1245 (1995).

${ }^{11}$ R. Riedel, A. Greiner, G. Miehe, W. Dreßler, H. Fueß, J. Bill, and F. Aldinger, Angew. Chem., Int. Ed. Engl. 36, 603 (1997).

${ }^{12}$ M. J. Cooper, Acta Crystallogr. 17, 1452 (1964).

${ }^{13}$ X. Liu, A. Decker, D. Schmitz, and R. Dronskowski, Z. Anorg. Allg. Chem. 626, 103 (2000).

${ }^{14}$ L. Stork, X. Liu, B. P. T. Fokwa, and R. Dronskowski, Z. Anorg. Allg. Chem. 633, 1339 (2007).

${ }^{15}$ F. P. Bowden and H. M. Montagu-Pollock, Nature (London) 191, 556 (1961).

${ }^{16}$ M. Becker, J. Nuss, and M. Jansen, Z. Naturforsch. 55b, 383 (2000).

${ }^{17}$ M. Becker and M. Jansen, Acta Crystallogr., Sect. C: Cryst. Struct. Commun. 57, 347 (2001).

${ }^{18}$ G. Baldinozzi, B. Malinowska, M. Rakib, and G. Durand, J. Mater. Chem. 12, 268 (2002).

${ }^{19}$ M. Becker and M. Jansen, Z. Anorg. Allg. Chem. 626, 1639 (2000).

${ }^{20}$ X. Liu, P. Müller, P. Kroll, and R. Dronskowski, Inorg. Chem. 41, 4259 (2002).

${ }^{21}$ X. Liu, P. Müller, and R. Dronskowski, Z. Anorg. Allg. Chem. 631, 1071 (2005)
${ }^{22}$ O. Reckeweg and F. J. DiSalvo, Z. Anorg. Allg. Chem. 629, 177 (2003).

${ }^{23}$ R. Srinivasan, M. Stöbele, and H.-J. Meyer, Inorg. Chem. 42, 3406 (2003).

${ }^{24}$ W. Liao, C. Hu, R. K. Kremer, and R. Dronskowski, Inorg. Chem. 43, 5884 (2004).

${ }^{25}$ M. Neukirch, S. Tragl, and H.-J. Meyer, Inorg. Chem. 45, 8188 (2006).

${ }^{26}$ M. Launay and R. Dronskowski, Z. Naturforsch. 60b, 437 (2005).

${ }^{27}$ X. Liu, M. Krott, P. Müller, C. Hu, H. Lueken, and R. Dronskowski, Inorg. Chem. 44, 3001 (2005).

${ }^{28}$ X. Liu, L. Stork, M. Speldrich, H. Lueken, and R. Dronskowski, Chem.-Eur. J. 15, 1558 (2009).

${ }^{29}$ M. Krott, X. Liu, B. P. T. Fokwa, M. Speldrich, H. Lueken, and R. Dronskowski, Inorg. Chem. 46, 2204 (2007).

${ }^{30}$ X. Liu, M. A. Wankeu, H. Lueken, and R. Dronskowski, Z. Naturforsch. 60b, 593 (2005).

${ }^{31}$ The minor reduction below the expectation $\mu$ value might be attributable by a small diamagnetic impurity due to leftover $\mathrm{Zn}^{2+}$ instead of $\mathrm{Mn}^{2+}$ cations; unfortunately, the metathesis reaction cannot be made $100 \%$ quantitative.

${ }^{32}$ J. Rodriguez-Carvajal, FULLPROF (version 3.2), Laboratoire Léon Brillouin, 1997.

${ }^{33}$ L. Corliss, N. Elliot, and J. Hastings, Phys. Rev. 104, 924 (1956).

${ }^{34}$ C. G. Shull, W. A. Strauser, and E. O. Wollan, Phys. Rev. 83, 333 (1951)

${ }^{35}$ W. L. Roth, Phys. Rev. 110, 1333 (1958).

${ }^{36}$ H. Shaked, J. Faber, Jr., and R. L. Hitterman, Phys. Rev. B 38, 11901 (1988).

${ }^{37}$ A. L. Goodwin, M. G. Tucker, M. T. Dove, and D. A. Keen, Phys. Rev. Lett. 96, 047209 (2006).

${ }^{38}$ R. A. Alikhanov, Zh. Eksp. Teor. Fiz. 36, 1690 (1959) [Sov. Phys. JETP 9, 1204 (1959)].

${ }^{39}$ A. S. Borovik-Romanov and M. P. Orlova, Sov. Phys. JETP 4, 531 (1957).

${ }^{40}$ J. E. Greedan, J. Mater. Chem. 11, 37 (2001). 